\title{
Nanocomposites with antibacterial properties using CNTs with magnetic nanoparticles
}

\author{
B. Ortega García ${ }^{1}$, O. V. Kharissova ${ }^{1}$, H. V. Rasika Dias ${ }^{2}$, \\ F. Servando Aguirre T. ${ }^{3}$, J. Salinas Hernández ${ }^{4}$ \\ ${ }^{1}$ Universidad Autónoma de Nuevo León, Facultad de Ciencias Físico-Matemáticas, \\ Monterrey, Nuevo León, México \\ ${ }^{2}$ The University of Texas at Arlington, Department Chemistry and Biochemistry, \\ Arlington, Texas 76019, USA \\ ${ }^{3}$ Centro de Investigación en Materiales Avanzados (CIMAV), \\ Monterrey, Nuevo León, México \\ ${ }^{4}$ Centro de Investigación y Desarrollo de Educación Bilingüe (CIDEB), \\ Monterrey, Nuevo León, México \\ beatriz.ortega24@gmail.com,okhariss@mail.ru,dias@exchange.uta.edu, \\ servando.aguirre@cimav.edu.mx, jeannie05@hotmail.com
}

\author{
PACS 81.07.-b, 81.07De, 82.35Cd, 82.35Np
}

DOI 10.17586/2220-8054-2016-7-1-161-168

In this work, multiwall carbon nanotubes (MWCNT) were functionalized with silver nanoparticles using two different methods of incorporation. Characterization of these composites was done using Raman spectroscopy and Transmission Electron Microscopy (TEM) and the antibacterial properties were measured using method of dilution and plating. The four-point probe method was used to measure the resistivity of the nanocomposite thin films made via the spin coating method.

Keywords: carbon nanotubes, functionalization, silver nanoparticles, polymers, nanocomposites.

Received: 20 November 2015

\section{Introduction}

It is well-known that diverse polymers possess antibacterial properties [1], however, this also applies to several nanoparticles (for example Ag-NPs [2]) or carbon nanotubes (CNTs) [3]. The CNTs can be magnetic if they contain iron nanoparticles inside as a result of the incorporation of Fe-containing catalysts during their production [4]. It is hoped that once the nanoparticles are integrated into the polymer matrix that all of the NPs properties will be transferred to the polymer, resulting in its antibacterial and magnetic properties. There are many applications reported for polymer-NP nanocomposites with antibacterial properties; however, in the case of also having magnetic properties, there also exists the potential for an alternative means for drug delivery or biosensor applications.

The natural polymer chitosan (CS) belongs to the group of antibacterial polymers [1]; it is compatible with tissues and is utilized in biomedicine. In this work, the functionalization of CNTs with $\mathrm{Ag}$ and $\mathrm{Fe}$ and their further uniform dispersion in chitosan polymeric matrix is studied. 


\section{Experimental}

\subsection{Materials}

Multiwall carbon nanotubes (MWCNTs) were prepared by spray pyrolysis method in the presence of iron as a catalyst at two different temperatures, $760{ }^{\circ} \mathrm{C}$ and $800{ }^{\circ} \mathrm{C}$. Chitosan (low molecular weight) from Aldrich, $\mathrm{AgNO}_{3}$ from Fisher, Luperox $\mathbb{R}$ LP, Lauroyl peroxide, $97 \%$ from Aldrich, Ethanol 99.2 \% from Decon Labs Inc., Acetic Acid (2 \%), cetyltrimethylammonium bromide (CTAB) from Aldrich, deionized water (DIW) were used as supplied.

\subsection{Functionalization of MWCNTs}

Two methods, used for functionalization of carbon nanotubes, were as follows:

For the first functionalization (1NP), the CNTs $(0.025 \mathrm{~g})$, synthesized at $800{ }^{\circ} \mathrm{C}$, were ultrasonically dispersed in ethanol $(10 \mathrm{~mL})$. Another solution containing DMF $(20 \mathrm{~mL})$, silver nanoparticles $(0.08 \mathrm{~g})$, prepared by a more environmentally-benign method, and chitosan ( 2 wt.\%), was added to the first solution under nitrogen flow and stirred for $48 \mathrm{hrs}$ (see Fig. 1.1).

For the second functionalization $(2 \mathrm{NP})$, the CNTs $(0.1 \mathrm{~g})$, synthesized at $760{ }^{\circ} \mathrm{C}$, were ultrasonically dispersed in ethanol $(400 \mathrm{~mL}$ ) and lauroyl peroxide (Luperox $\mathbb{R}$ LP, Lauroyl peroxide) $(0.5 \mathrm{~g})$ was added for $30 \mathrm{~min}$. Next, the CTAB $(0.2 \mathrm{~g})$ was added and the mixture was subjected to ultrasonication for $15 \mathrm{~min}$. After this step, one of previously selected silver compounds (silver(I) acetate, silver(II) oxide or $\mathrm{AgNO}_{3}$ ) was added and ultrasonication was continued for an additional $60 \mathrm{~min}$.
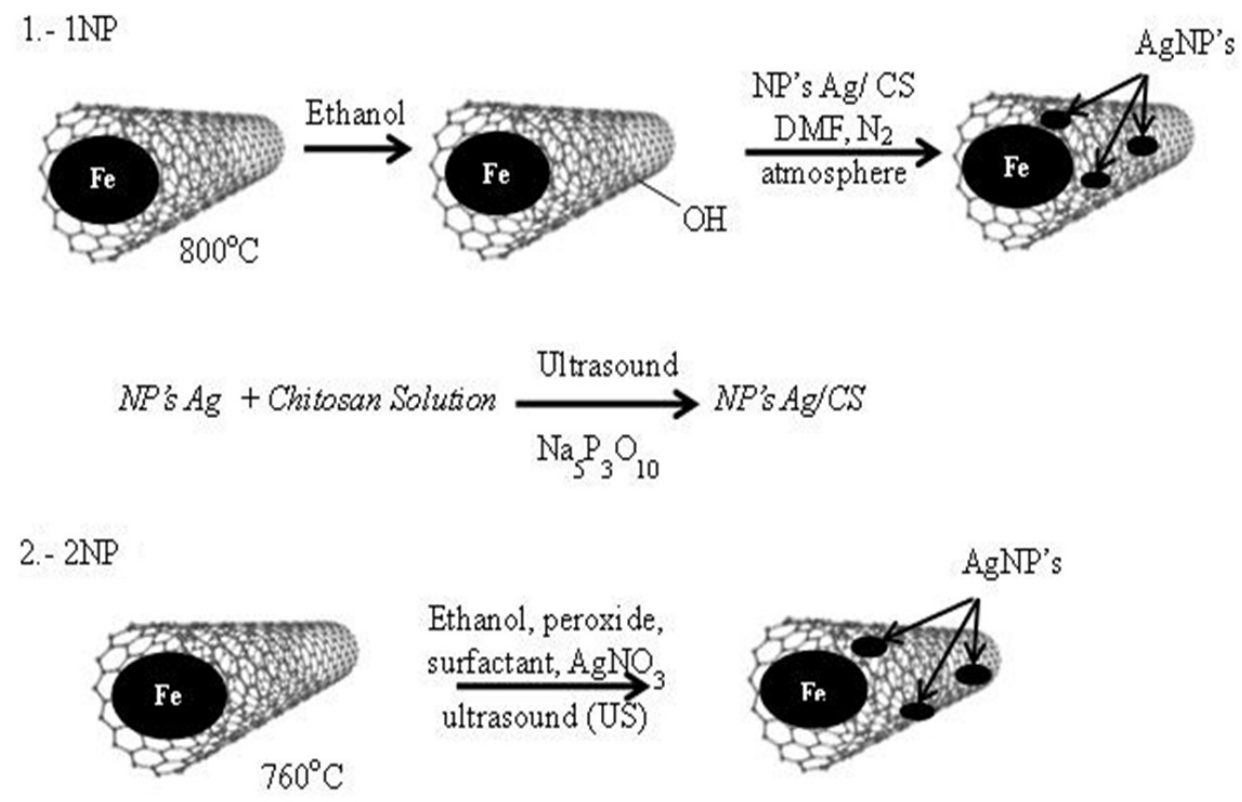

FIG. 1. Two methods for carbon nanotube functionalization with silver nanoparticles

\subsection{Preparation of CS/Ag/MWCNT nanocomposite}

Carbon nanotubes functionalized with silver nanoparticles were then used $(0.02 \%$ wt.\%) for the preparation of nanocomposites with different chitosan contents $(1 \%, 3 \%$ and $5 \%)$ by mixing using ultrasonication for 1 and $5 \mathrm{~min}$. Films of different nanocomposites were obtained by a spin coating technique onto glass slides. 


\subsection{Characterization}

The samples were analyzed by Raman spectroscopy, Transmission Electron Microscopy (TEM); method of dilution and plating was used for measurement of antibacterial property. The four-point probe method was applied to measure the resistivity of the nanocomposites.

\subsubsection{Method of dilution and plating:}

1. A colony of the strain Escherichia coli DH5a was placed in $75 \mathrm{~mL}$ of liquid nutrient medium for $12 \mathrm{~h}$ at $37^{\circ} \mathrm{C}$ (pre-culture).

2. The analyzed samples were Luperox or Lauroyl peroxides, CTAB, mixture of peroxide and $\mathrm{CTAB}$, ethanol, silver nitrate solution as a reference, carbon nanotubes synthesized at $760{ }^{\circ} \mathrm{C}$ and carbon nanotubes containing silver nanoparticles. All reagents were introduced into an ultrasonic bath for 5 min for sample dispersion.

3. $1 \mathrm{~mL}$ of the pre-culture of $E$. coli $\mathrm{DH} 5$ a was mixed in $15-20 \mathrm{~mL}$ of melted nutrient medium agar which was cooled to $40{ }^{\circ} \mathrm{C}$, and allowed to solidify undisturbed on a flat table top.

4. $0.1 \mathrm{~mL}$ of the dispersed samples were taken and poured over the filter paper disks and placed in the center of the Petri dish with the bacteria, for this process, sterilization of equipment was done using a Bunsen burner (called "disk technique").

5. $0.05 \mathrm{~mL}$ of the scattered samples were taken and poured into a hole made in the center of the agar plate placed in the center of the petri dish with the bacteria, for this process, sterilization of equipment was done using a Bunsen burner (called agar hole technique)

6. Both plates (paper disks and agar holes) were maintained at $37^{\circ} \mathrm{C}$ for $12 \mathrm{~h}$.

Note. The Luperox and CTAB were diluted in absolute ethanol and later dispersed by ultrasonic treatment for $25 \mathrm{~min}$.

\section{Results and discussions}

Using Raman spectroscopy and TEM analyses, we confirmed the functionalization of the synthesized carbon nanotubes $\left(760{ }^{\circ} \mathrm{C}\right)$. Fig. 2 shows the Raman spectra of MWCNT synthetized at $760{ }^{\circ} \mathrm{C}$ and two characteristic peaks are observed: one peak at a Raman shift of $1310 \mathrm{~cm}^{-1}$, called the D-band, which is assigned to the disordered graphitic structure of MWCNTs. The other peak, at a Raman shift of $1600 \mathrm{~cm}^{-1}$, called the G-band, is assigned to the $\mathrm{C}=\mathrm{C}$ bond in the graphitic plane.

The Raman spectra of both pristine MWCNT and MWCNT/Ag, obtained with an excitation 284 energy of $2.41 \mathrm{eV}$, are shown in Fig. 2 (an inset in this image corresponds to the reported data for SWCNTs/Ag [5]). The details of sample preparation and Raman experiment have been reported elsewhere [5]. The presence of silver in the Raman spectra is confirmed by the presence of characteristic peaks in the $1100-1600 \mathrm{~cm}^{-1}$ range. These peaks are slightly shifted and broadened in comparison to the Raman data of the samples without silver. It should be pointed out that the tangential band (at about $1590 \mathrm{~cm}^{-1}$ ) is composed of several peaks. The downshift in the tangential $\mathrm{G}^{+}$-mode from 1600 to $1593 \mathrm{~cm}^{-1}$ indicates that electrons are transferred from the silver to the MWCNTs; a similar peak amplification was previously reported for SWCNT/Ag [5].

Figure 3 shows the TEM images of MWCNTs synthesized at $760{ }^{\circ} \mathrm{C}$ and MWCNT with silver nanoparticles. Carbon nanotubes synthesized using an iron-containing precursor and functionalized with silver nanoparticles demonstrate magnetic properties. As it is seen in Fig. 4 using a small magnet, the nanotubes are attracted to the magnet. This confirms that magnetic properties are not lost upon functionalization. 


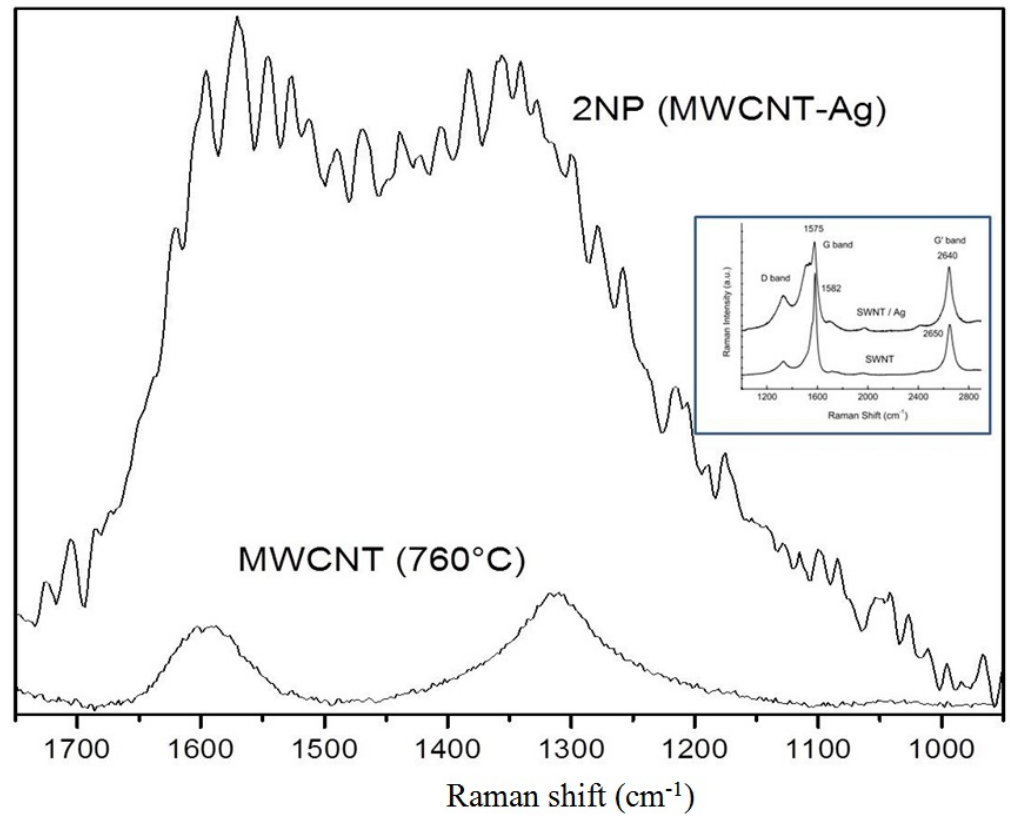

FIG. 2. Raman spectra of MWCNT: Raman spectra of MWCNTs made at $760{ }^{\circ} \mathrm{C}$ with Raman spectra of functionalized MWCNTs made at $760{ }^{\circ} \mathrm{C}$ and silver nanoparticles. An inset contains earlier reported data for SWCNTs/Ag
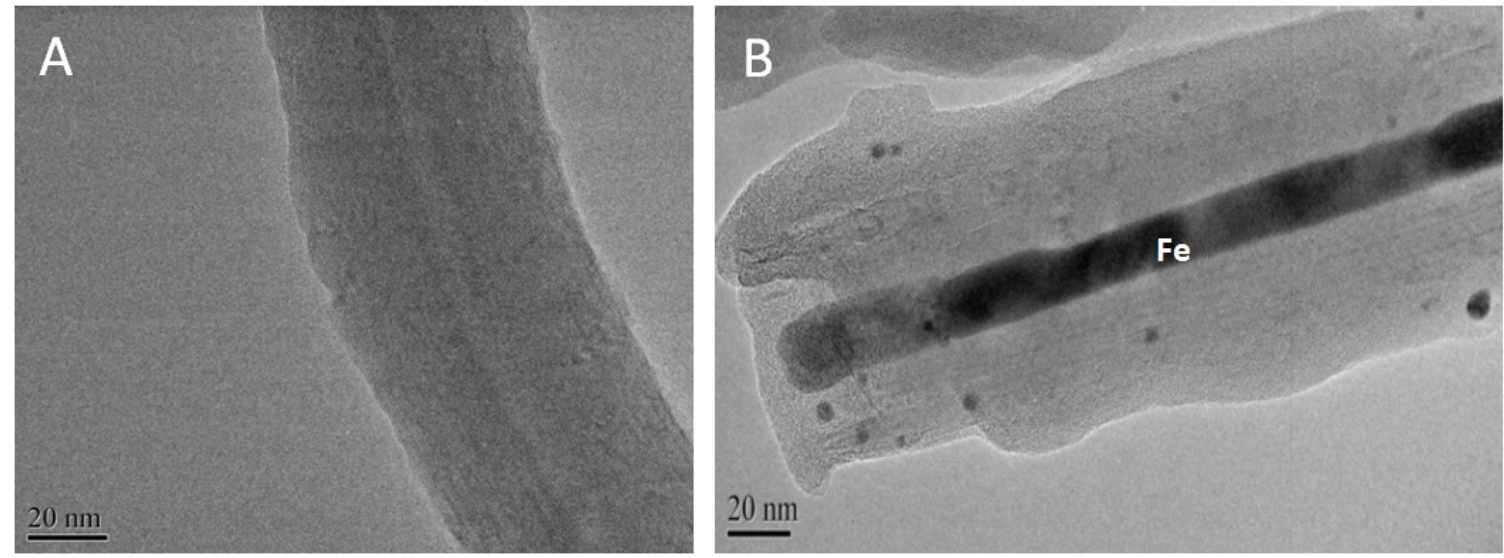

FIG. 3. TEM micrograph: A) MWCNT made at $760^{\circ} \mathrm{C}$ and $\mathrm{B}$ ) functionalized MWCNT $\left(760^{\circ} \mathrm{C}\right)$ with silver nanoparticles $(2 \mathrm{NP})$

Figure 5 and Fig. 9 show an increase in the resistivity when you increase the number of layers using chitosan solution for $1 \%$ and $3 \%$ and $1 \mathrm{NC}$ and 2NC. In Fig. 7, high contents of CS (5\%) were used; one layer presented high resistivity to infinity but increasing other layer resistivity is decreased. In case of addition of nanoparticles (1NC and $2 \mathrm{NC}$ ), only with $2 \mathrm{NC}$ the similar decrease of resistivity takes place. of CS.

Figures 8 and 9 show a decrease of resistivity when there is an increase of percentage

\subsection{Antibacterial test of carbon nanotubes with silver}

The CNT samples with silver nanoparticles at two concentrations $(0.0199 \mathrm{~g} / 0.5 \mathrm{~mL}$ and $0.00199 \mathrm{~g} / 10 \mathrm{~mL}$ ) were observed to generate homogeneous foams, while for the remaining 


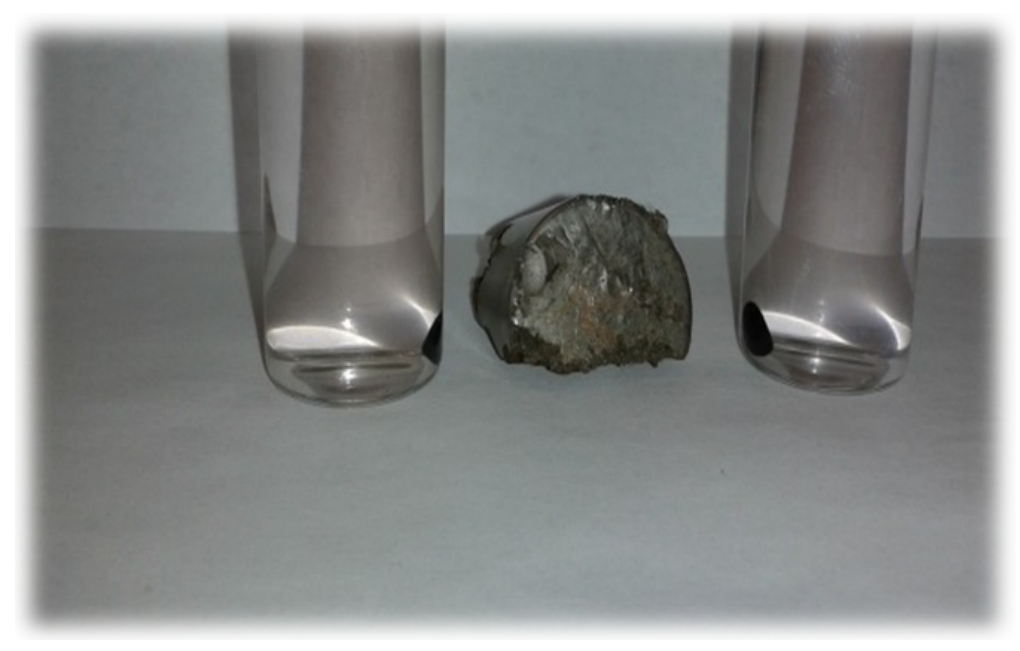

FIG. 4. Carbon nanotubes with magnet nearby

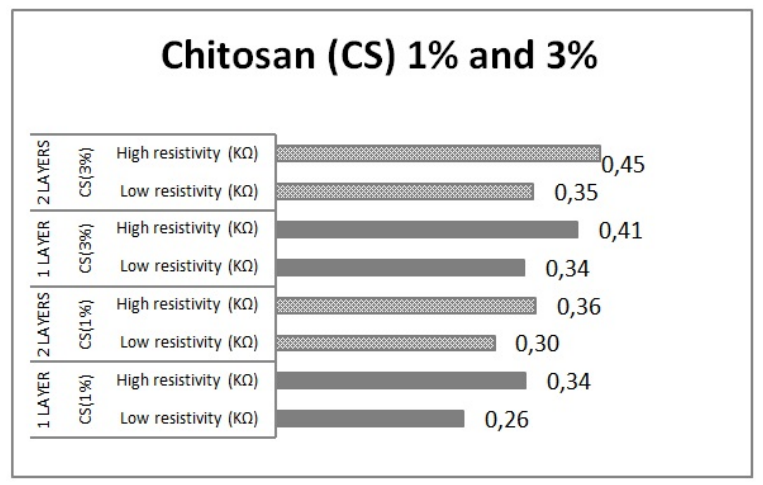

FIG. 5. Results for solutions with different percentages of chitosan

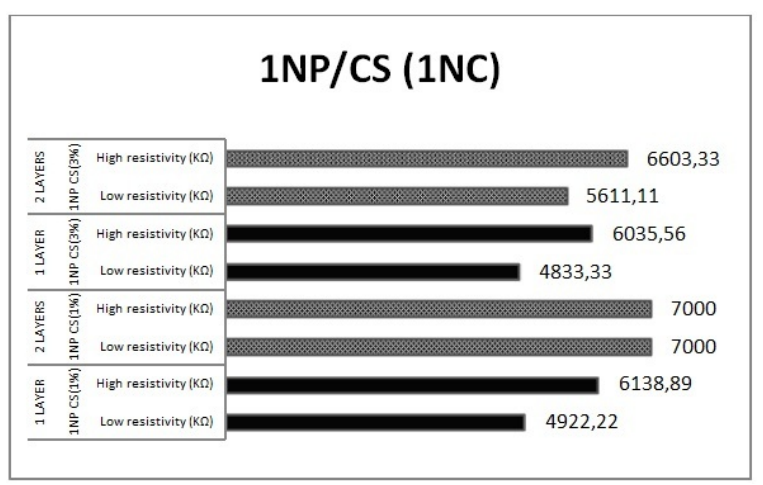

FIG. 7. Decrease in resistivity average $(\mathrm{M} \Omega)$ of $1 \mathrm{NC}$ nanocomposite with $\mathrm{CS}$ solution $(1 \%$ and $3 \%)$

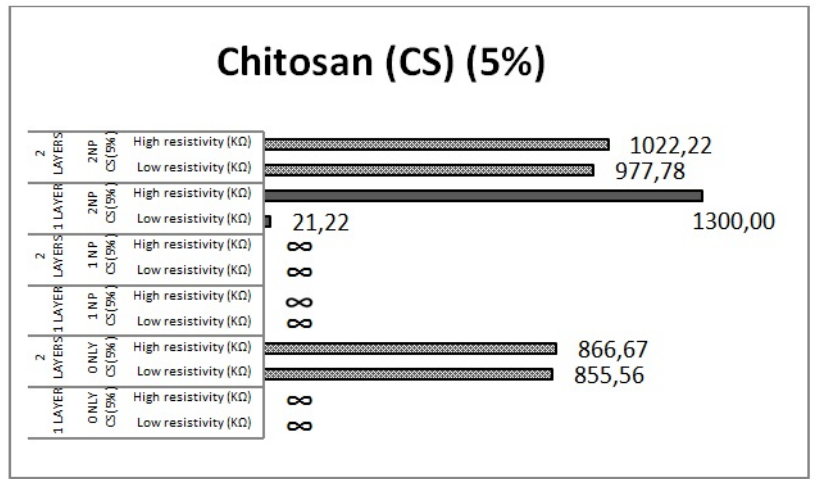

FIG. 6. Results for chitosan solution of $5 \%$ using only chitosan and two different nanoparticles (1NP and 2NP)

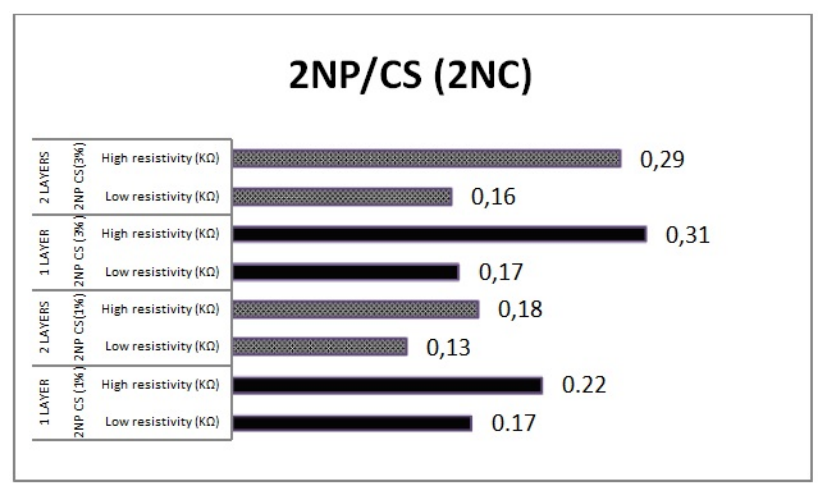

FIG. 8. Decrease in resistivity average $(\mathrm{K} \Omega)$ of $2 \mathrm{NC}$ nanocomposite with $\mathrm{CS}$ solution $(1 \%$ and $3 \%)$ 


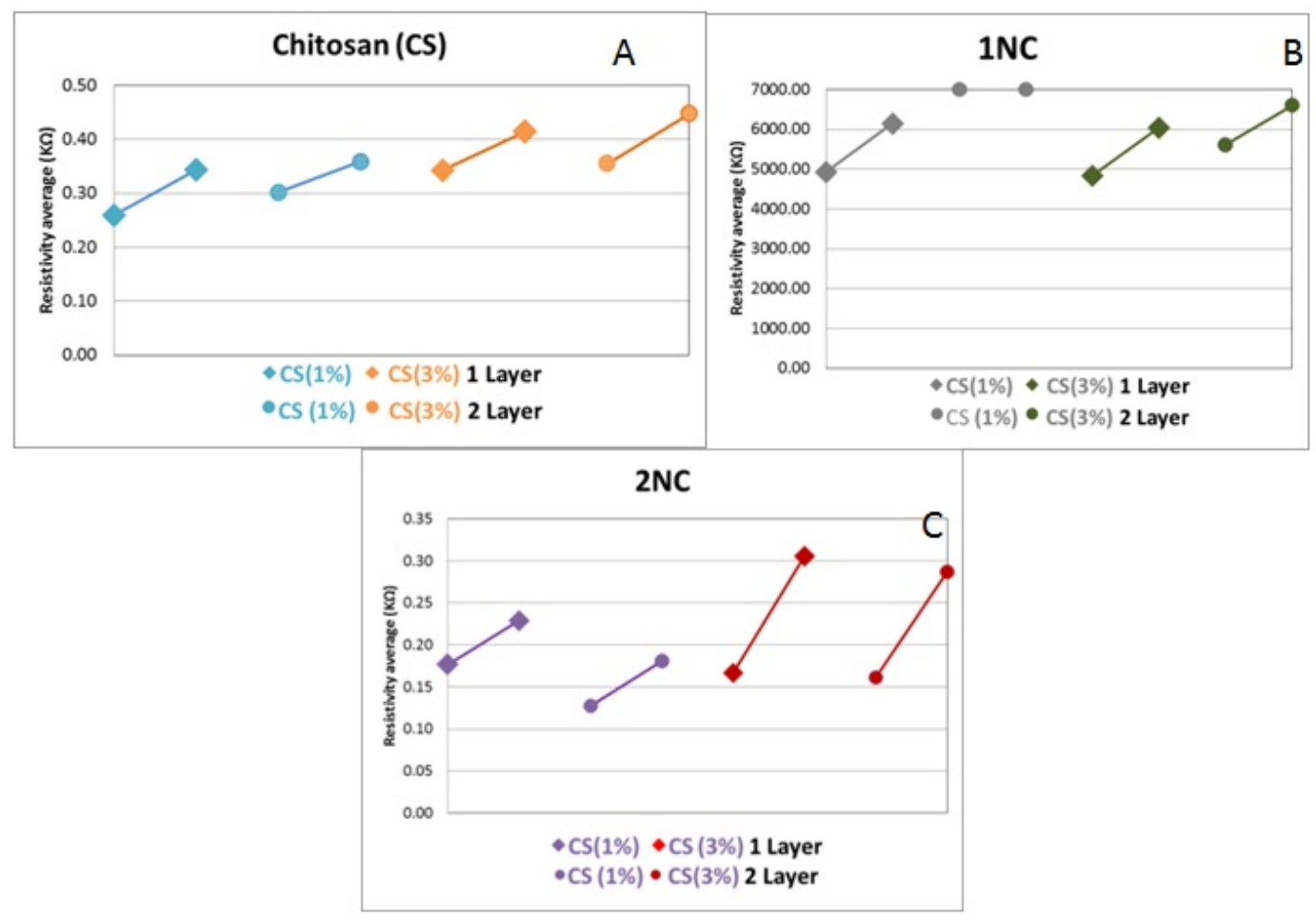

FIG. 9. Resistivity average for 1 layer and 2 layers of A) CS solution (1\% and $3 \%$ ) and nanocomposites B) $1 \mathrm{NC}$ and C) $2 \mathrm{NC}$

samples, dispersion was difficult. Because this sample showed foaming due to the surfactant CTAB used in the reaction, an experimental design was utilized whereby all reagents were tested in order to rule out that the growth inhibitory activity of Escherichia coli DH5 was attributable to the effect of one of them.

The CTAB in agar hole in petri dish showed an inhibition halo of $3.0 \mathrm{~cm}$ of diameter. The CTAB in paper filter disk showed in petri dish an inhibition halo of $0.1 \mathrm{~cm}$ of diameter.

In Fig. 10, the sample with a concentration of $0.02 \mathrm{~g} / 0.5 \mathrm{~mL}$ in $0.05 \mathrm{~mL}$ poured in the agar holes of the sample MWCNT/Ag showed an inhibition halo of $2.3 \mathrm{~cm}$ of diameter in the $E$. coli DH5a culture. The samples with a concentration of $0.02 \mathrm{~g} / 0.5 \mathrm{~mL}$ in $0.1 \mathrm{~mL}$ in disks, only the sample MWCNT/Ag showed an inhibition halo of $0.5 \mathrm{~cm}$ of diameter in the $E$. coli DH5a culture (see Table 1).

\section{Conclusion}

In the present work, we obtained nanoparticles with antibacterial and magnetic properties using an ultrasonic method for functionalization. We confirm the functionalization with Raman analysis by comparing the Raman spectra of MWCNT with and without functionalization. In addition to the antibacterial and magnetic properties of our nanoparticles, we measured the resistivity of chitosan and the nanocomposites. One of the nanocomposites (2NC) decreased the resistivity among $33 \%$ (1 layer) and $58 \%$ (2 layers) less for CS (1\%) and $36 \%$ (1 layer) and $46.6 \%$ (2 layers) less for CS (3\%). These mean that multiwall carbon nanotubes with silver nanoparticles allow increasing the conductivity of the nanocomposite. 
Nanocomposites with antibacterial properties...

TABLE 1. Antimicrobial growth inhibition

\begin{tabular}{|c|c|c|c|}
\hline \multirow{2}{*}{ Sample } & \multicolumn{3}{|c|}{ Antimicrobial growth inhibition } \\
\hline & Technique: & Agar hole & Disk \\
\hline Control (only agar with bacteria) & NO & & \\
\hline Peroxide & NO & & \\
\hline CTAB & YES & & \\
\hline Mix of peroxide and CTAB & NO & & \\
\hline Ethanol & NO & & \\
\hline CNT & NO & $*$ & \\
\hline CNT with Ag & YES & & \\
\hline
\end{tabular}




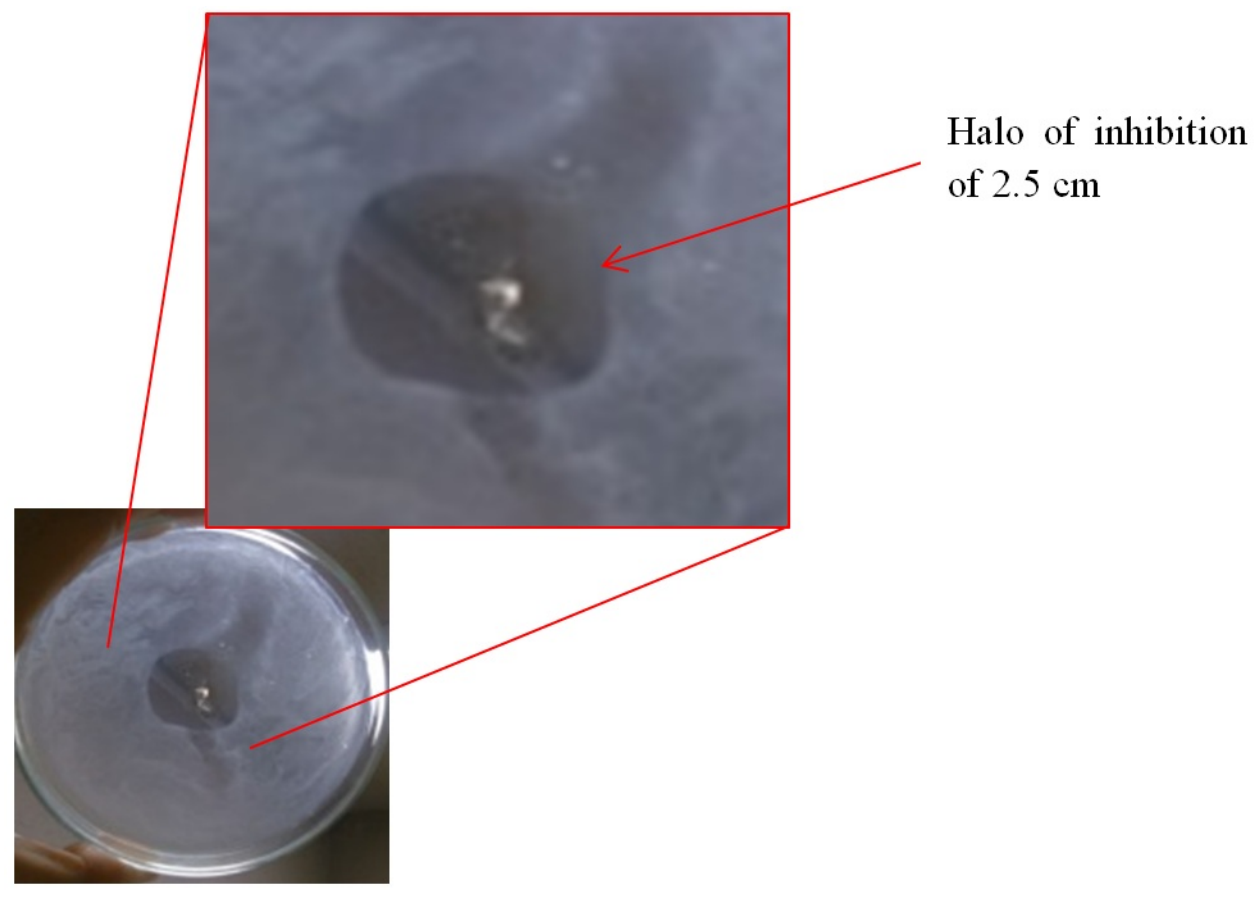

FIG. 10. MWCNT with silver nanoparticles (2NP) with a concentration of 0.02 $\mathrm{g} / 0.5 \mathrm{~mL}$ in $0.05 \mathrm{~mL}$ poured in the agar holes with a halo of inhibition of 2.3 $\mathrm{cm}$

\section{Acknowledgements}

This work was supported by CONACYT and Universidad Autónoma de Nuevo León, and also we are grateful to UTA, CIMAV and CIDEB for their collaboration.

\section{References}

[1] Muñoz-Bonilla A., Fernández-García M. Polymeric materials with antimicrobial activity. Progr. Polymer Sci., 2012, 37, P. 281-339.

[2] Simchi A., Tamjid E., Pishbin F., Boccaccini A.R. Recent progress in inorganic and composite coatings with bactericidal capability for orthopaedic applications. Nanomedicine: Nanotechnology, Biology, and Medicine, 2011, 7, P. 22-39.

[3] Dastjerdi R., Montazer M. A review on the application of inorganic nano-structured materials in the modification of textiles: Focus on anti-microbial properties. Coll. Surf. B: Biointerfaces, 2010, 79, P. 5-18.

[4] Gui X., Wei J., Wang K., Wang W., Lv R., Chang J., Kang F., Gu J., Wu D. Improved filling rate and enhanced magnetic properties of Fe-filled carbon nanotubes by annealing and magnetic separation. Mater. Res. Bull., 2008, 43, P. 3441-3446.

[5] Fagan S.B., Souza Filho A.G., Mendes Filho J., Corio P., Dresselhaus M.S. Electronic properties of Ag-and CrO 3-filled single-wall carbon nanotubes. Chem. Phys. Lett., 2005, 406(1), P. 54-59. 